\title{
Fertilización sintética y orgánica y su efecto en la producción de maíz, variedad Nutrinta Amarillo
}

\section{Fertilization synthetic and organic and effect on the production of maize variety yield Nutrinta}

\begin{abstract}
Miguel Jerónimo Ríos ${ }^{1}$, Jorge Gómez-Martínez ${ }^{2}$, Ricardo Eliezer Bolaños Aguilar ${ }^{3}$, Carlos José Gutiérrez Matamoros ${ }^{3}$
${ }^{1}$ Especialista en fertilidad de suelos, Docente-Investigador, ORCID: https// orcid.org/0000-0002-6664-5317, mrios@ci.una.edu.ni/2MSc. en agroecología y desarrollo sostenible, Docente-Investigador, ORCID: https://orcid.org/0000-0002-4157-4874, Jorge.gomez@ci.una.edu.ni $/{ }^{3}$ Ingeniero Agrónomo
\end{abstract} Universidad Nacional Agraria

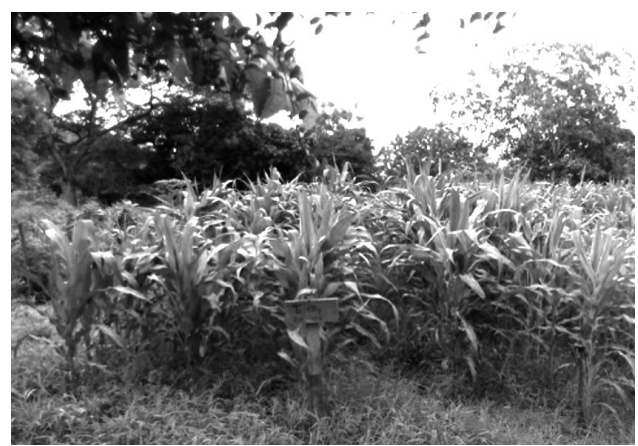

\section{RESUMEN}

En Nicaragua el maíz (Zea mays L.) ocupa un lugar importante en la economía, siendo considerado el producto de mayor consumo en la alimentación humana. Con este estudio se pretende generar información necesaria acerca de nuevas fórmulas de fertilizantes (Nitro xtend y Fertimaíz) respondiendo a la problemática de los agricultores en sus bajos rendimientos. El trabajo de investigación fue realizado en el centro de experimentación y validación de tecnología (CEVT) Las Mercedes, ubicado en el km 11 Carretera Norte, Managua, Nicaragua y entre las coordenadas geográficas de $12^{\circ} 10^{\prime} 14^{\prime \prime}$ y $12^{\circ} 08^{\prime} 05^{\prime}$ " de latitud Norte y $86^{\circ} 10^{\prime} 22^{\prime \prime}$ y $86^{\circ} 09^{\prime} 44^{\prime \prime}$ de longitud Oeste a una altura de $56 \mathrm{msnm}$. Se realizó durante los meses de octubre 2015 a enero 2016. Los suelos de esta zona son derivados de cenizas volcánicas, de la serie Las Mercedes, de textura franco arcillosa. El objetivo fue comparar el efecto que tiene las fuentes de fertilizantes sintéticos y orgánicos sobre el crecimiento y rendimiento del cultivo de maíz. Se estableció en arreglo unifactorial en diseño de bloques completo al azar con cuatro repeticiones y tres tratamientos, distribuidos de la siguiente forma: el tratamiento uno corresponde a la apliación de fertilizante tradicional (12-30-10 más urea), el segundo tratamiento fue fertilizante especial (Fertimaiz y Nitro xtend) y el tercer tratamiento corresponde a un orgánico (humus de lombriz). La dimensión del ensayo fue de $410.8 \mathrm{~m}^{2}$. Los datos fueron analizados con el programa INFOSTAT y los resultados indican diferencia significativa en la variable: diámetro del tallo $(2.15 \mathrm{~cm})$, altura de inserción de la espiga $(225.08 \mathrm{~cm})$, presentando el tratamiento tradicional los mejores resultados; las variables, altura de planta $(215.43 \mathrm{~m})$, numero de hojas (8.58), área foliar (604.06 $\left.\mathrm{cm}^{2}\right)$. Inserción de la espiga $(129.77 \mathrm{~cm})$, rendimiento $(3453.48 \mathrm{~kg}$ $\mathrm{ha}^{-1}$ ) los mejores resultados se reflejaron al fertilizar con Fertimaiz y Nitro Xtend.

Palabras clave: fertilización especial, Fertimaiz, Nitro Xtend, rendimiento, humus.

Recibido: 16 de octubre del 2018 Aceptado: 21 de marzo del 2019

\section{ABSTRACT}

In Nicaragua, the corn (Zea mays L) production occupies an important place in the economy, being considered the product of greater human consumption and main source of calories. This study pretends to generate necessary information about the fertilization formulas (Nitro xtend and Fertimaíz) responding to the farmer problems of their low maize yields. The research was conducted in the center of experimentation and validation of technology (CEVAT), Las Mercedes located at km 11 Carretera Norte, Managua Nicaragua with geographic coordinates from $12^{\circ} 10^{\prime} 14^{\prime \prime}$ to $12^{\circ} 08^{\prime} 05^{\prime}$ North latitude and $86^{\circ} 10^{\prime} 22^{\prime \prime}$ to $86^{\circ} 09^{\prime} 44^{\prime \prime}$ West longitude, at 56 meters above see level, carried out during the months of October 2015 to January 2016. The soils in this area are derived from volcanic ash, young soils and it belongs to the Las Mercedes Serie of clay loam texture. The aim was to compare the effect of different sources of synthetic and organic fertilizers on the growth and yield of maize, the first with traditional fertilizer (12-30-10 and urea), the second with special fertilizers (fertimaiz and nitro xtend) and organic (humus). The total area of the assay was $410.8 \mathrm{~m}^{2}$ and data were analyzed with the INFOSTAT satatistical program. The the results show that there were significant differences in the variables: stalk diameter $(2.15 \mathrm{~cm})$, height of insertion of the tassel $(225.08 \mathrm{~cm})$, the traditional treatment showed the best results, with plant height $(215.43 \mathrm{~m})$, number of leaves $(8.58)$, leaf area $(604.06 \mathrm{~cm} 2)$. But, Tassel insertion $\left(129.77 \mathrm{~cm}\right.$ ) and yield (3 $453.48 \mathrm{~kg} \mathrm{ha}^{-1}$ were better with the treatment of Fertimaiz y Nitro Xtend.

Keywords: Fertilization especial, Fertimaiz, Nitro Xtend, yield, humus. 


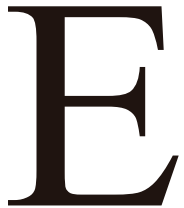

maíz (Zae mays L.) ha formado parte de la dieta de los países de América Latina y África, por ser una de las especias de mayor adaptación en cuanto a condiciones ambientales, mayor producción y múltiples usos, ya sea como alimento humano, animal y fuente de materia prima en la agroindustria. En el 2001 se produjo cerca de 600 millones de toneladas por año nivel mundial y se visualizó que había una población mundial de 6000 000, y que para el año 2030 habrá un aumento del 33\% de la población, por lo que se necesitaran 60 millones de toneladas adicionales para satisfacer la demanda de este cereal (FAO, 2001).

El maíz es uno de los principales cultivos a nivel nacional y tiene una relevancia en términos económicos y social para el país por su uso en la alimentación humana y como insumo en la actividad, avícola y porcina. En el caso del maíz amarillo, en Nicaragua se estima una superficie de producción entre 7026 y 10539 hectáreas; esta variedad puede utilizarse durante tres ciclos de siembra sin tener disminución significativa en el rendimiento, los que oscilan entre 2910 y $3881 \mathrm{~kg} \mathrm{ha}^{-1}\left(2045\right.$ y $\left.2727 \mathrm{~kg} \mathrm{mz}^{-1}\right)$, rendimientos superiores al obtenido con variedades criollas (Acevedo, 2005).

La variedad Nutrinta Amarillo proviene de la población S99TLYQ-AB y fue introducida por el Programa Regional de Maíz para Centroamérica y el Caribe (PRM) y el Centro Internacional de Mejoramiento de Maíz y Trigo (CIMMYT) en convenios de colaboración con el Programa Nacional de Maíz de Nicaragua. La variedad Nutrinta Amarillo fue desarrollada por el Proyecto de Investigación y Desarrollo del Instituto Nicaragüense de Tecnología Agropecuaria (INTA). Instancia que en el 2011 indica que la variedad fue validada en 33 localidades de Nicaragua.

El maíz blanco es de baja calidad debido a que la proteína principal del grano (zeina) es deficiente de lisina y triptófano, en cambio la variedad nutrinta amarillo contiene el doble de proteína que el maíz blanco, lo que brinda una buena alternativa para alimentación de aves y cerdos. El INTA (2011) reporta que la mayor ganancia diarias de peso en cerdo es $\left(0.694 \mathrm{~kg} \mathrm{día}^{-1}\right)$

con el suministro de maíz amarillo, lo que representa un aumento del $26 \%$ en relación al peso ganado con maíz blanco (0.55 kg día-1), además indica, que posee mayor porcentaje de triptófano $(10.4 \%)$, un aminoácido esencial para el crecimiento del ser humano.
Para obtener resultados óptimos en cuanto a producción, se debe de tomar en cuenta, el tipo de semilla a utilizar; las condiciones edafoclimaticas, dosis y tipo de fertilizante; es por ello que un mal manejo de la fertilidad del suelo, pilar fundamental en el aprovechamiento de los sistemas agrícolas, conlleva a bajos niveles de rendimiento, lo que se traduce en un mal llenado de granos, pocos granos, mazorcas pequeñas, etc. Por eso un plan de fertilización impacta de manera favorable en los resultados técnico-económicos de la empresa, y es fundamental que exista un proceso de planificación, dentro del cual se deberá definir un plan de fertilización (Melgar y Torres, 2007).

El estudio pretende generar información necesaria acerca de la efectividad de nuevas fórmulas comerciales de fertilizantes (Nitro Xtend y Fertimaíz) que DISAGRO, ofertan al mercado, respondiendo a la problemática de los agricultores en cuanto a sus bajos rendimientos, mala calidad del grano y altos costos de producción, obtenidos con el uso tradicional de fertilizantes (completo 12-30-10 y urea 46\%), además de comparar el efecto del fertilizante orgánico (humus de lombriz) con respecto a los sintéticos.

\section{MATERIALES Y MÉTODOS}

Ubicación del ensayo. El estudio se realizó en la hacienda Las Mercedes propiedad de la Universidad Nacional Agraria, ubicada en el km 11 Carretera Norte, entrada al CARNIC $800 \mathrm{~m}$ al Norte. Sus coordenadas geográficas corresponden a $12^{\circ} 10^{\prime} 14^{\prime \prime}$ a $12^{\circ} 08^{\prime} 05^{\prime \prime}$ de latitud Norte y $86^{\circ} 10^{\prime} 22^{\prime \prime}$ a $86^{\circ} 09^{\prime} 44^{\prime \prime}$ de longitud Oeste, a $56 \mathrm{msnm}$ (Lino y Flores, 2015). El ensayo se estableció en los meses de octubre del 2015 y enero del 2016, y las evaluaciones se realizaron cada siete días a partir del 15 de octubre.

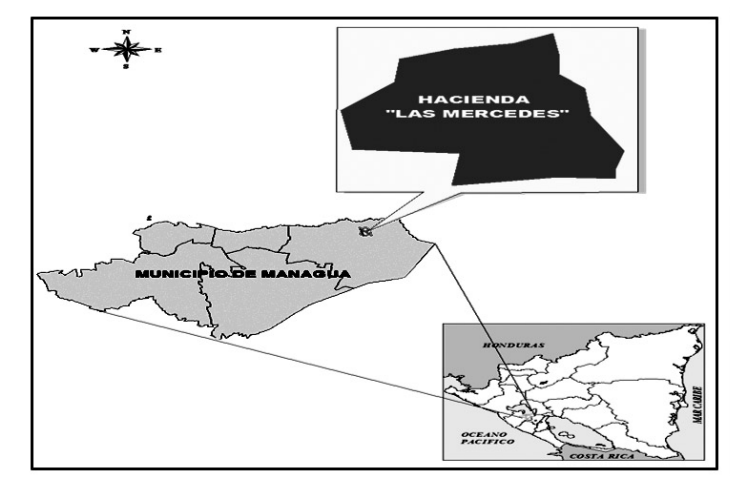

Figura 1. Ubicación geográfica del centro de experimentación y validación de Tecnología, Las Mercedes.

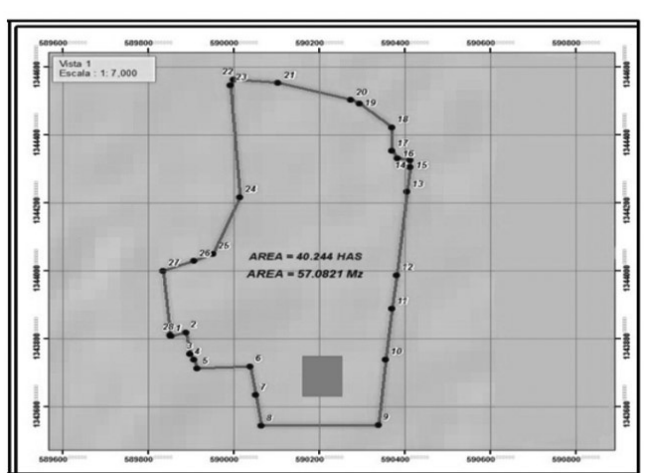

Clima. La temperatura durante el período de estudio varió entre 22.9 y $30.5^{\circ} \mathrm{C}$, con una precipitación media de 1134 $\mathrm{mm}$, humedad relativa de $71.5 \%$ y velocidad máxima del viento de $4.23 \mathrm{~m} \mathrm{~s}^{-1}$. 
Suelo. Según Villanueva (1990) el suelo donde se realizó el experimento pertenece a la serie Las Mercedes derivados de cenizas volcánicas catalogado como franco arcilloso de orden Inceptisol. Son suelos jóvenes pocos evolucionados que presentan capas endurecidas que conduce a lo que se traduce como perfiles con diferentes secuencias texturales, otras subunidades del suelo tienen mal drenaje, pero también existen otros que son adecuadamente drenados; estos suelos contienen alto contenido de potasio. Las propiedades químicas del mismo se presentan en el siguiente cuadro.

Cuadro 1. Análisis químico del suelo finca Las Mercedes, 2014

\begin{tabular}{cccccc}
\hline $\mathrm{pH}$ & $\begin{array}{c}\mathrm{MO} \\
(\%)\end{array}$ & $\begin{array}{c}\mathrm{N} \\
(\%)\end{array}$ & $\begin{array}{c}\mathrm{P} \\
(\mathrm{ppm})\end{array}$ & $\begin{array}{c}\mathrm{K} \\
(\mathrm{Meq} / 100 \mathrm{~g})\end{array}$ & $\begin{array}{c}\text { Prof muest } \\
(\mathrm{cm})\end{array}$ \\
\hline \multirow{2}{*}{6.82} & 3.8 & $\begin{array}{c}0.19 \\
\mathrm{M}\end{array}$ & $\begin{array}{c}3.9 \\
\mathrm{~B}\end{array}$ & $\begin{array}{c}4.19 \\
\mathrm{~A}\end{array}$ & 25 \\
& $\mathrm{M}$ & $\mathrm{M}$ & & \\
\end{tabular}

A: Alto M: Medio, B: Bajo

Fuente: Laboratorio de suelos y agua UNA (2014).

Diseño experimental. El ensayo se estableció en arreglo unifactorial en diseño de Bloques Completos al Azar (BCA), con cuatro repeticiones y tres tratamientos. El área total del experimento fue de $410.8 \mathrm{~m}^{2}$, y una superficie por bloque de $83.20 \mathrm{~m}^{2}$. Los tratamientos estuvieron formados por 5 surcos de 8 metros de longitud y $0.8 \mathrm{~m}$ para un total de $25.6 \mathrm{~m}^{2} \mathrm{de}$ unidad experimental. Cabe señalar que de los cinco surcos se tomaron los dos centrales como parcela útil, considerando el efecto de borde. Los tratamientos evaluados se describen en el Cuadro 2.

Cuadro 2. Descripción de los tratamientos

\begin{tabular}{llc}
\hline Tratamientos & Formula & Dosis $\left(\mathrm{kg} \mathrm{ha}^{-1}\right)$ \\
\hline $\mathrm{T}_{1}$ Fertilizantes & Completo 12-30-10 & 282 \\
tradicionales & Urea 46 \% & 220 \\
$\mathrm{~T}_{2}$ Fertilizantes & Fertimaíz 18-20-10-1.5-7.2-1.2 & 140 \\
especiales & Nitro Xtend 46-0-0 & 163 \\
$\mathrm{~T}_{3}$ Humus de & $2.22 \mathrm{~N}-0.88 \mathrm{P}-0.64 \mathrm{~K}$ & 6099 \\
lombriz & & \\
\hline
\end{tabular}

Descripción de la variedad. En el ensayo se utilizó maíz amarillo variedad Nutrinta liberada por el INTA. Esta variedad se puede sembrar desde los 200 a los $1000 \mathrm{~m}$ de altura, se adapta a suelos francos, franco arenoso y areno arcilloso, con pendientes desde $15 \%$ hasta más de $30 \%$, pH de 6.5-7.0, temperaturas $22-29^{\circ} \mathrm{C}$ y precipitaciones durante el ciclo biológico del cultivo de 1000 a $1800 \mathrm{~mm}$. Las características agronómicas que presenta esta variedad son: Días a flor femenina de 54 a 56, Altura de plantas de 220 a 230 centímetros, Altura de inserción de mazorca de 110 a 120 centímetros, textura de grano semi cristalino, color de grano amarrillo, días a cosecha 110 a 115 , madurez relativa intermedia, rendimiento comercial de 45 a $60 \mathrm{qq}$ $\mathrm{mz}^{-1}\left(2911-3881 \mathrm{~kg} \mathrm{ha}^{-1}\right)$ (INTA, 2004).
Manejo agronómico

Preparación de suelo. La preparación del suelo se efectuó el día veintiséis de septiembre del 2015, de forma mecanizada, mediante el método de labranza convencional, consistió en la limpia del terreno, un pase de arado y un pase de grada.

Siembra. La siembra se realizó a chorrío, después de la germinación se hizo el raleo dejando una distancia de siembra de $0.30 \mathrm{~m}$ entre planta y $0.80 \mathrm{~m}$ entre surco.

Riego. El sistema de riego fue por aspersión con frecuencia de dos horas diarias.

Fertilización. La fertilización se realizó sobre la base del análisis químico de suelo. Después de realizar los respectivos cálculos se pesaron las porciones necesarias de los fertilizantes para cada tratamiento. La fertilización se realizó al momento de la siembra y en el caso de los portadores independientes de nitrógeno, se fraccionaron a los 15 y 30 días después de la siembra (urea $46 \%$ y Nitro Xtend 46\%).

Manejo fitosanitario. Se realizó un monitoreo o registro semanalmente para identificar cuáles son las principales insectos y patógenos asociados a este cultivo, y de esta manera tomar algunas medidas de manejo, aplicando Basillus thuringiensis en 40 g por bombada (20 1) como preventivo y luego se realizó la aplicación del insecticida winner (Spinosyn) a razón de $5 \mathrm{ml}$ por bombada de 20 litros para el manejo de Spodoptera sp.

Control de malezas. El manejo de maleza se realizó a los 18, 36,54 y 72 días después de la siembra de forma manual con machete y azadón.

\section{Variables evaluadas}

Variables de crecimiento

Altura de la planta (cm). Se medió la altura de la planta desde el nivel del suelo hasta la base de la yema de la hoja bandera, mediante el uso de una cinta métrica, en diez plantas al azar con intervalos de siete días.

Diámetro del tallo $(\mathbf{m m})$. Se realizó con un vernier en el entrenudo de la parte media del tallo en diez plantas al azar a intervalos de siete días.

Número de hojas. Se contabilizaron las hojas activas y sin daño mecánico en diez plantas seleccionadas al azar a intervalos de siete días.

Área foliar $\left(\mathbf{c m}^{2}\right)$. Se obtuvo a partir de la multiplicación de la longitud y el ancho central de la hoja por la constante 0.73 correspondiente al cultivo de maíz CYMMIT (2008). 
Altura de inserción de la mazorca (cm). Se registró desde el nivel del suelo hasta la base de la panoja en diez plantas al azar.

Altura a la inserción de la espiga (panoja) $\mathrm{cm}$. Se midieron desde el nivel del suelo hasta la inserción de la espiga.

Longitud de la espiga (panoja). Se midieron desde la inserción de la espiga hasta su ápice.

Rendimiento $\mathrm{kg} \mathrm{ha}^{-1}$. Se pesó lo cosechado en cada parcela útil y se convirtió a $\mathrm{kg} \mathrm{ha}^{-1}$.

Análisis estadístico. Se realizó un análisis de varianza (ANDEVA) y separación de medias a través de la prueba de rangos múltiples de Duncan al 5\% usando el programa INFOSTAT versión 2016.

Cuadro 3. Altura de la planta $(\mathrm{cm})$ a partir de los 21 días después de la siembra

\begin{tabular}{lccccccc}
\hline Tratamiento & $21 \mathrm{dds}$ & $28 \mathrm{dds}$ & $35 \mathrm{dds}$ & $42 \mathrm{dds}$ & $49 \mathrm{dds}$ & $56 \mathrm{dds}$ & $63 \mathrm{dds}$ \\
\hline Fertilizante tradicional & 28.26 & 32.50 & $90.86 \mathrm{a}$ & $133.95 \mathrm{a}$ & $171.18 \mathrm{a}$ & $196.91 \mathrm{a}$ & $212.24 \mathrm{a}$ \\
Fertilizante especial & 27.55 & 29.73 & $91.10 \mathrm{a}$ & $133.97 \mathrm{a}$ & $176.48 \mathrm{a}$ & $201.05 \mathrm{a}$ & $215.43 \mathrm{a}$ \\
Humus de lombriz & 24.99 & 29.88 & $57.00 \mathrm{~b}$ & $84.46 \mathrm{~b}$ & $119.66 \mathrm{~b}$ & $146.39 \mathrm{~b}$ & $158.72 \mathrm{~b}$ \\
\hline CV $(\%)$ & 10.43 & 26.45 & 13.94 & 9.56 & 15.36 & 14.56 & 10.92 \\
\hline$p \leq 0.05$ & 0.2453 & 0.8657 & 0.0073 & 0.0011 & 0.0280 & 0.0470 & 0.0160 \\
\hline
\end{tabular}

dds: días después de la siembra.

Indicadores de eficiencia de uso de nutrientes. Estos indicadores se obtuvieron a partir del nitrógeno, debido a que las dosis usadas en el cultivo son calculadas en base a este elemento.

Los indicadores evaluados corresponden a:

PPF: Productividad parcial del factor

$\mathrm{PPF}=\frac{\text { Rendimiento } \mathrm{kg}}{\text { cantidad de nutriente aplicado }}$

EA: Eficiencia agronómica EA Rendimiento kg con nutriente - rendimiento kg sin nutriente cantidad de nutriente aplicado

Para los indicadores existe un rango a nivel de la región de Centroamérica y México (International Plant Nutrition Institute, 2013). Para el indicador productividad parcial del factor, el rango en nitrógeno varía de 40 a 80 y para el indicador eficiencia agronómica el rango en nitrógeno varia de 10-30.

\section{RESULTADOS Y DISCUSIÓN}

\section{Variables de crecimiento}

Altura de la planta. Greulach y Adams (1980), definen crecimiento como el incremento en la cantidad de protoplasma en un organismo, notable por el aumento irreversible en talla y peso, implicando la división y agrandamiento de las células, tejidos y órganos; además de ello Orozco, (1996), menciona que la altura de planta es el resultado de la elongación del tallo, al acumular nutrientes producidos en la fotosíntesis.

En el Cuadro 3 se indica que a los 21 y 28 días después de la siembra no existe diferencia significativa y que a los 35, 42, 49, 56 y 63 días después de la siembra se presentan diferencia altamente significativa, siendo el tratamiento especial (Fertimaíz + Nitro Xtend) el que presentó las mayores alturas. Esto se debe a las características de este fertilizante ya que presenta menores pérdidas de nitrógeno por volatilización, además tiene la función de inhibir la acción de la ureasa. Los valores presentados en este estudio a los 63 días $(215.43 \mathrm{~cm})$ son similares a las reportadas por INTA (2014) y por Lino y Flores (2015), los cuales reportan alturas de (217 $\mathrm{cm})$ y (217.61) respectivamente.

Diámetro del tallo. El diámetro del tallo es un parámetro de gran importancia en la planta de maíz, ya que influye sobre el acame y el rendimiento (García y Watson, 2003).

En el Cuadro 4 se presenta el comportamiento del diámetro del tallo a partir de los 21 días después de la siembra y se aprecia que no hay diferencia significativa a los 21 y 28 días, pero sí a los 35 y 42 días después de la siembra, superando los fertilizantes sintéticos al orgánico. Según el INTA (2001) la aplicación de nitrógeno es uno de los factores que influye en el diámetro de las plantas. Camacho y Bonilla (1999), aseguran que el diámetro del tallo aumenta a medida que el nivel de nitrógeno aumenta y a medida que el cultivo se desarrolla demanda más nutriente, es en este punto donde el fertilizante sintético entra en contacto con la humedad del suelo y se produce una hidratación rápida y los nutrientes están disponible para la planta al momento de la fertilización. Los valores presentados en este estudio fueron inferiores a los reportados por Sobalvarro y Díaz (2016), quienes registraron diámetros de tallo de hasta $2.46 \mathrm{~cm}$.

Cuadro 4. Diámetro del tallo $(\mathrm{cm})$ a partir de los 21 días después de la siembra

\begin{tabular}{lcccc}
\hline Tratamientos & $21 \mathrm{dds}$ & $28 \mathrm{dds}$ & $35 \mathrm{dds}$ & $42 \mathrm{dds}$ \\
\hline Fertilizante tradicional & 0.93 & 1.58 & $1.98 \mathrm{a}$ & $2.15 \mathrm{a}$ \\
Fertilizante especial & 0.90 & 1.42 & $1.96 \mathrm{a}$ & $2.09 \mathrm{a}$ \\
Humus de lombriz & 0.81 & 1.37 & $1.38 \mathrm{~b}$ & $1.63 \mathrm{~b}$ \\
\hline $\mathrm{CV}(\%)$ & 13.20 & 26.86 & 13.35 & 4.82 \\
\hline$p \leq 0.05$ & 0.3835 & 0.7416 & 0.0188 & 0.0005
\end{tabular}

dds: Días después de la siembra. 
Número de hojas. A medida que la planta crece se pueden perder de tres a cinco hojas debido a la falta de nutrientes, engrosamiento del tallo, alargamiento de entrenudos y enfermedades foliares (Somarriba, 1998).

A los 21 y 28 días después de la siembra no se presentaron diferencias significativas, pero sí a los 35 y 42 días después de la siembra, donde la separación de medias por Duncan establece dos categorías agrupando a los tratamientos sintéticos (Fertilización tradicional y fertilización especial) en una sola categoría. Con el humus de lombriz se registró menor número de hojas por planta (Cuadro 5).

Los valores del humus de lombriz se deben a que en las primeras etapas del cultivo el humus no sustenta la demanda de nutrientes, aunque con el tiempo mejora las propiedades físicas, químicas y biológicas del suelo.

Los resultados registrados fueron inferiores al compararlo con el estudio realizado por Flores y Lino (2015), que obtuvieron un promedio 9.48 hojas por plantas usando una fertilización tradicional.

Cuadro 5. Número de hojas por planta a partir de los 21 días después de la siembra

\begin{tabular}{lcccc}
\hline Tratamientos & $21 \mathrm{dds}$ & $28 \mathrm{dds}$ & $35 \mathrm{dds}$ & $42 \mathrm{dds}$ \\
\hline Fertilizante Tradicional & 4.33 & 6.60 & $7.53 \mathrm{a}$ & $8.43 \mathrm{a}$ \\
Fertilizante Especial & 4.40 & 6.85 & $7.88 \mathrm{a}$ & $8.58 \mathrm{a}$ \\
Humus de lombriz & 4.30 & 6.43 & $6.53 \mathrm{~b}$ & $7.23 \mathrm{~b}$ \\
\hline $\mathrm{CV}(\%)$ & 7.51 & 7.14 & 6.40 & 5.29 \\
\hline$p \leq 0.05$ & 0.9074 & 0.4858 & 0.0157 & 0.0080 \\
\hline
\end{tabular}

dds: Días después de la siembra.

Área foliar. El área foliar es una manifestación cuantitativa de las plantas que puede ser medido a través de ciertos parámetros tales como: ancho de hoja, longitud de la hoja y número de nudos (Tapia y Camacho, 1988).

A los 35 y 42 días después de la siembra se registran diferencias estadísticas superando al fertilizante orgánico los tratamientos sintéticos.

Vázquez (1999), afirma que el área foliar es un parámetro de importancia en la evaluación del crecimiento de las plantas, de ahí la importancia de la captación de la radiación fotosintética, la cual permite la translocación de foto asimilados al grano, de igual manera la FAO (2001), asegura que el índice de área foliar es importante para determinar la intercepción de la radiación solar en la planta de maíz.

Flores y Lino (2015) encontraron medias de 702.05 $\mathrm{cm}^{2}$ y Sobalvarro y Díaz (2016), registraron medias de 647.89 $\mathrm{cm}^{2}$, en ambos estudios el área foliar fue superior a los obtenidos en este experimento $\left(604.06 \mathrm{~cm}^{2}\right)$ (Cuadro 6).
Altura de inserción de la mazorca. Cantarero y Martínez (2002), refieren que el carácter inserción de mazorca es de importancia al momento de seleccionar una variedad para la producción de grano, sin embargo, no existe una altura definida para este carácter, además a mayor altura de inserción de mazorca, la planta tendrá más hojas que la proveerán de nutrientes y por ende mayor rendimiento del cultivo.

Se registran diferencias altamente significativas, separando en dos categorías; una a los fertilizantes sintéticos y la otra al fertilizante orgánico (cuadro 7); estos resultados fueron superiores a los reportados por Sobalvarro y Díaz (2016) que encontraron promedios de 60.88 para el tratamiento especial.

Según la empresa DISAGRO (2011), el fertilizante especial (Nitro Xtend) es un fertilizante que además de incorporar los elementos necesarios (nitrógeno, fosforo y potasio) también contiene zinc, azufre, y magnesio elementos esenciales en los procesos fisiológicos de la planta, además Nitro Xtend, reduce las pérdidas de nitrógeno por volatilización.

Altura de inserción de la espiga (panoja). La espiga o panoja es la emergencia de la inflorescencia masculina a través del cogollo, luego de la emergencia total de la panoja ocurre la antesis, que se define como la aparición de las anteras de las flores en las espiguillas de la panoja (Marthin, 2016).

Se registran diferencias significativas y dos categorías estadísticas, ubicando el tratamiento humus de lombriz con el menor promedio (Cuadro 7).

Ruda (2010) evaluó diferentes fechas de siembra en el rendimiento y calidad del maíz usando humus de lombriz, y registró menor altura con la aplicación de humus de lombriz.

Acevedo (2005) indica que en la etapa $V_{10}$ (40-60 días después de la siembra) se desarrolla rápidamente la panoja y los entrenudos de la planta, ya que en esta etapa habrá 
mayor absorción de biomasa, Nitrógeno, fosforo, Potasio y agua, es por ello que es importante la nutrición especialmente de macroelementos que sean aprovechados por la planta.

Longitud de la espiga (panoja). Para esta variable no se registran diferencias estadísticas (Cuadro 7).

Cuadro 7. Alturas de inserción de Mazorca, inserción de espiga (panoja) y espiga (panoja)

\begin{tabular}{lccc}
\hline Tratamiento & $\begin{array}{c}\text { altura de inserción } \\
\text { de mazorca }(\mathrm{cm})\end{array}$ & $\begin{array}{c}\text { altura de inserción } \\
\text { de espiga }(\mathrm{cm})\end{array}$ & $\begin{array}{c}\text { Longitud de } \\
\text { espiga }(\mathrm{cm})\end{array}$ \\
\hline Fertilizante Tradicional & $125.96 \mathrm{a}$ & $225.08 \mathrm{a}$ & 36.23 \\
Fertilizante Especial & $129.77 \mathrm{a}$ & $220.20 \mathrm{a}$ & 35.44 \\
Humus de lombriz & $90.36 \mathrm{~b}$ & $173.98 \mathrm{~b}$ & 36.60 \\
\hline $\mathrm{CV}(\%)$ & 10.73 & 8.83 & 7.21 \\
\hline$p \leq 0.05$ & 0.0075 & 0.0136 & 0.8187 \\
\hline
\end{tabular}

Rendimiento en $\mathbf{~ g ~ h a}^{-1}$. Los mejores rendimientos se obtienen con la aplicación de los fertilizantes sintéticos. Según el INTA (2013), los rendimientos aproximados de la variedad nutrinta amarillo oscila entre 2900 y $3200 \mathrm{~kg} \mathrm{ha}^{-1}$, el cual se ve reflejado en los resultados obtenidos en este estudio (Figura 2).

Los rendimientos registrados con el fertilizante especial (3 $453.48 \mathrm{~kg} \mathrm{ha}^{-1}$ ) fueron similares a los obtenidos por Sobalvarro y Díaz (2016) quienes obtuvieron rendimientos de $3937.5 \mathrm{~kg} \mathrm{ha}^{-1}$ para el tratamiento especial; los resultados de estos estudios demuestran que las nuevas fórmulas ( $\mathrm{Ni}$ tro Xtend y Fertimaíz) disminuyen las pérdidas de nitrógeno, aumentan la disponibilidad de nutrientes y proporcionan mejores beneficios para la planta por ser de liberación lenta, y además, estas formulas poseen moléculas que están recubiertas con azufre y permanecen más tiempo disponibles para la planta (International Plant Nutrition Institute, 2013).

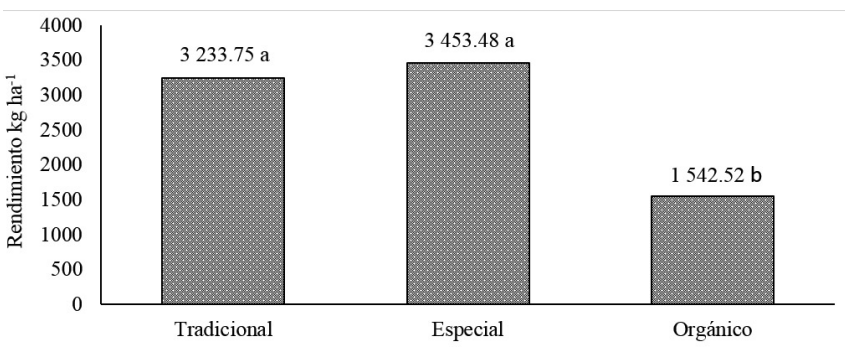

Figura 2. Rendimiento del cultivo de Maíz $\left(\mathrm{kg} \mathrm{ha}^{-1}\right)$, en los tratamientos evaluados, finca las Mercedes, Managua 2015.

Determinación de dos indicadores de eficiencia de uso de nutrientes. La evaluación de los indicadores de eficiencia del uso de nutrientes se determina por el método de diferencia, simple, eficiente y adecuado para investigaciones en fincas, este método también consiste en la diferencia en rendimiento del cultivo o toma de nutrientes entre parcelas fertilizadas y no fertilizadas (International Plant Nutrition Institute 2005).
Indicadores evaluados. La mayoría de los ecosistemas naturales y agrícolas, al ser fertilizados con nitrógeno inorgánico, muestran importantes incrementos en la productividad, poniendo en evidencia la importancia de este elemento (Pereyra, 2011).

Productividad parcial del factor (PPF). La productividad parcial del factor nos indica que tan productivo es el sistema de producción considerado con relación a la cantidad de nutriente aplicado (International Plant Nutrition Institute 2005). Los valores obtenidos en este estudio fueron menores a las referencias (40 a $80 \mathrm{~kg}$ maíz $/ \mathrm{kg} \mathrm{N}$ ), por lo que se podría decir que la productividad en las dos estrategias de fertilización fue baja.

$$
\begin{gathered}
\text { PPFt1N }=\frac{3233.75 \mathrm{~kg} \mathrm{ha}^{-1}}{135.39 \mathrm{~kg} \mathrm{~N} \mathrm{ha}^{-1}}=23.88 \mathrm{~kg} \text { maiz kg N } \\
\text { PPFt2N }=\frac{3453.48 \mathrm{~kg} \mathrm{ha}^{-1}}{100.18 \mathrm{~kg} \mathrm{~N} \mathrm{ha}^{-1}}=34.47 \mathrm{~kg} \text { maíz kg N } \\
\text { PPFt3N }=\frac{1542.52 \mathrm{~kg} \mathrm{ha}^{-1}}{135 \mathrm{~kg} \mathrm{Nha}^{-1}}=11 \mathrm{~kg} \text { maíz kg N }
\end{gathered}
$$

Eficiencia agronómica (EA). La eficiencia agronómica indica cuanto se ganó en productividad por usar este nutriente (International Plant Nutrition Institute 2005). Para el indicador eficiencia agronómica el rango en nitrógeno varia de 10$30 \mathrm{~kg}$ maíz $\mathrm{kg}^{-1}$ nutriente. Según los cálculos obtenidos, en el caso de las aplicaciones de los fertilizantes sintéticos, los valores se encuentran dentro del rango $(12.85-19.56)$, lo que indica que existió una ganancia adecuada en productividad por el uso del nitrógeno proveniente de estos fertilizantes y su disponibilidad para la planta.

Para el cálculo de eficiencia agronómica se tomó como referencia el historial de producción de las parcelas que no han sido fertilizadas y que han registrado un rendimiento de $1493.37 \mathrm{~kg}$ maíz ha ${ }^{-1}$ registrado por Sobalvarro y Díaz (2016).

$$
\begin{gathered}
\text { EAt1N }=\frac{3233.75 \mathrm{~kg} \mathrm{ha}^{-1}-1493.37 \mathrm{~kg} \text { maíz ha-1 }}{135.39 \mathrm{~kg} \mathrm{~N} \mathrm{ha}^{-1}}=12.85 \mathrm{~kg} \text { maíz kg N } \\
\text { EAt2N }=\frac{3453.48 \mathrm{~kg} \mathrm{ha}^{-1}-1493.37 \mathrm{~kg} \mathrm{ha}^{-1}}{100.18 \mathrm{~kg} \mathrm{~N} \mathrm{ha}^{-1}}=19.56 \mathrm{~kg} \text { maíz kg N } \\
\text { EAt2N }=\frac{1542.52 \mathrm{~kg} \mathrm{ha}^{-1}-1493.37 \mathrm{~kg} \mathrm{ha}^{-1}}{135 \mathrm{~kg} \mathrm{~N} \mathrm{ha}^{-1}}=0.36 \mathrm{~kg} \text { maíz kg N }
\end{gathered}
$$

Según los resultados obtenidos el tratamiento especial se ubicó en primer lugar, seguido del tradicional el cual 
se encontró en un rango óptimo, sin embargo, el tratamiento a base de humus de lombriz, estuvo muy por debajo de los datos permitidos.

\section{CONCLUSIONES}

Todas las variables, excepto longitud de espiga, registraron mayores valores por efecto del fertilizante especial (Nitro
Xtend y Fertimaíz) y el fertilizante tradicional a base de completo de la fórmula $12-30-10$ y urea al $46 \%$.

Se registró una productividad parcial del factor (PPF) baja, en comparación a la referencia internacional y, una eficiencia agronómica (EA) adecuada en productividad, por el uso del nitrógeno proveniente de los fertilizantes sintéticos.

\section{REFERENCIAS BIBLIOGRÁFICAS}

Acevedo, E. (2005). Fisiología del rendimiento Maíz. Universidad de Chile. Santiago-Chile, 5.

Camacho García, J., \& Bonilla Abad, R. (1999). Efecto de tres niveles de nitrógeno y tres densidades poblacionales sobre el crecimiento, desarrollo y rendimiento en el cultivo de maiz (Zea mays L.) Var. NB-6 (Doctoral dissertation, Universidad Nacional Agraria, UNA). Cantarero, R., \& Martínez, O. (2002). Evaluación de tres tipos de fertilizantes (gallinaza, estiércol vacuno y un fertilizante mineral) en el cultivo de maíz (Zea mays L) variedad NB-6. Trabajo de Diploma. Managua Nicaragua. Universidad Nacional Agraria Facultad de Agronomía.

CIMMYT (Centro Internacional para el Mejoramiento del Maíz y el Trigo). 1988. La formulación de recomendaciones a partir de datos Económicos. Un manual metodológico de evolución económica. ME.DF. Cimmyt.79 p.

(disagro, 2011) Espinoza, A. (2011). Variedad de maíz Nutrinta amarillo. Managua, NI. INTA (Instituto Nicaragüense de Tecnología Agropecuaria, NI), CNIA (Centro Nacional de Investigación Agropecuaria, NI).

Flores Arias, H. P., Frank, L., \& Melvin, J. (2015). Eficiencia de dos tipos de fertilizantes sintéticos en el crecimiento y rendimiento del cultivo de maiz (Zea mays L.) variedad Nutrinta amarillo, Centro Experimental Las Mercedes 2014 (Doctoral dissertation, Universidad Nacional Agraria).

García, M., \& Watson, C. (2003). Herencia de la resistencia al acame de raíces en maíz dulce (Zea mays L.). Revista Científica UDO Agrícola, 3(1), 24-33.

Greulach, V., \& Adams, J. E. (1980). Laa plantas, introducción a la botánica moderna. Edit. Limusa. p, 349-350.

INTA. (20 de feb de 2011). www.funica.org.ni/docs/granbasic_33pdf. Obtenido de www.funica.org.ni/docs/granbasic_33pdf: www. funica.org.ni/docs/granbasic_33pdf

InfoStat, 2009. InfoStat, versión 2009. Manual del Usuario. Grupo InfoStat, FCA, Universidad Nacional de Córdoba. Primera Edición, Editorial Brujas Argentina. 334 p.

INTERNATIONAL PLANT NUTRITION INSTITUTE (IPNI). 2005. Conozca y resuelva los problemas del maíz. (IPNI). (En línea). ES, consultado 21 enero. 2013, disponible en http://www.ipni.net/ppiweb/ltamn.nsf/\$webindex/article=85FA0405052570C8004DEFDFA79AD75D

INTA. (Instituto Nicaragüense de Tecnología Agropecuaria) 2013.Catalogo de semillas de granos básicos. Variedades de arroz, sorgo y maíz liberadas (en línea). Managua, NI. 74P. Consultado 18 feb 2016 Disponible en:http://www.observatorioredsicta.info/pdf_files/ catalogoSemillasInta.pdf

INTA (Instituto Nicaragüense de Tecnología Agropecuaria)2014. Variedad mejorada nutrinta amarillo maíz de alta calidad de proteína 3 p. Consultado 20 feb. 2016. Disponible en: http://www.funica.org.ni/docs/gran_basic_33.pdf

INTA (Instituto Nicaragüense de Tecnología Agropecuaria) 2004 Variedad mejorada nutrinta amarillo maíz de alta calidad de proteína 3 p. Consultado 20 feb. 2016. Disponible en: http://www.funica.org.ni/docs/gran_basic_33.pdf

Marthin, O. G. (10 de abril de 2016). http://ecathasl. s3. amazonaws. com/forrajicultura/cultivomaiz.pdf. Obtenido de http://ecathasl. s3. amazonaws. com/forrajicultura/cultivomaiz.pdf: http://ecathasl. s3. amazonaws. com/forrajicultura/cultivomaiz.pdf

Melgar, R, Torres; Torres, (18 de feb de 2016). Manejo de la fertilización en Maíz. Proyecto Fertilizar INTA Obtenido de. http://www. biblioteca.org.ar/libros/210722.pdf.

Orozco Calero, M. E. (1996). Efecto de tres niveles de gallinaza en plantaciones de cacao (Theobroma cacao L.) en desarrollo (Doctoral dissertation, Universidad Nacional Agraria, UNA

Pereyra, C. 2001. (12 abr. 2016) Asimilación de Nitrógeno en plantas AR. Argentina. en línea: http://exa.unne.edu.ar/biologia/fisiologia. vegetal /Asimilacion\%20del\%20nitrogeno.pdf

Ruda Vega, M. (10abr 2010) Efectos de la Fecha de Siembra sobre el Rendimiento y Calidad Comercial del Maíz Dulce (Zea mays L., var. saccharata körn) obtenido de http://ri.agro.uba.ar/files/download/tesis/especializacion/2011 rudavegamarcelo.pdf.

Sobalvarro, Y; Díaz, E.2016. Comparación de dos fórmulas comerciales en el cultivo de maíz (Zea mays L.) Cultivar Nutrinta amarillo, centro de experimentación y validación de tecnología las Mercedes. (Ingenieria Universidad Nacional Agraria, UNA).

Somarriba R., C. (1998). Texto granos básicos, Managua, universidad nacional agraria

Tapia, B.H y Camacho, A. (1988). Control integrado de la producción de frijol común Basado en cero labranza Managua, G.T.Z.

Vásquez, H, V. (1999) Î́ndice de área foliar, acumulación de materia seca y rendimiento de granos de maíz bajo tres condiciones de agua en el suelo, Coahuila, México.

Villanueva, E. (1990). Los suelos de la finca Las Mercedes y las propiedades Más relevantes para planear su uso y manejo. (ingeniería. Universidad Nacional Agraria. UNA). 